\title{
Correction to: Genome-enabled insights into the biology of thrips as crop pests
}

\author{
Dorith Rotenberg ${ }^{1 *}$, Aaron A. Baumann ${ }^{2}$, Sulley Ben-Mahmoud ${ }^{3}$, Olivier Christiaens ${ }^{4}$, Wannes Dermauw ${ }^{4}$, \\ Panagiotis loannidis ${ }^{5,6}$, Chris G. C. Jacobs ${ }^{7}$, Iris M. Vargas Jentzsch' ${ }^{8}$, Jonathan E. Oliver ${ }^{9}$, Monica F. Poelchau ${ }^{10}$, \\ Swapna Priya Rajarapu', Derek J. Schneweis ${ }^{11}$, Simon Snoeck ${ }^{12,4}$, Clauvis N. T. Taning ${ }^{4}$, Dong Wei ${ }^{4,13,14}$, \\ Shirani M. K. Widana Gamage ${ }^{15}$, Daniel S. T. Hughes ${ }^{16}$, Shwetha C. Murali ${ }^{16}$, Samuel T. Bailey ${ }^{17}$,
} Nicolas E. Bejerman ${ }^{18}$, Christopher J. Holmes ${ }^{17}$, Emily C. Jennings ${ }^{17}$, Andrew J. Rosendale ${ }^{17,19}$, Andrew Rosselot ${ }^{17}$, Kaylee Hervey ${ }^{11}$, Brandi A. Schneweis ${ }^{11}$, Sammy Cheng ${ }^{20}$, Christopher Childers ${ }^{10}$, Felipe A. Simão ${ }^{6}$, Ralf G. Dietzgen ${ }^{21}$, Hsu Chao ${ }^{16}$, Huyen Dinh ${ }^{16}$, Harsha Vardhan Doddapaneni ${ }^{16}$, Shannon Dugan ${ }^{16}$, Yi Han ${ }^{16}$, Sandra L. Lee ${ }^{16}$, Donna M. Muzny ${ }^{16}$, Jiaxin Qu ${ }^{16}$, Kim C. Worley $^{16}$, Joshua B. Benoit ${ }^{17}$, Markus Friedrich ${ }^{22}$, Jeffery W. Jones ${ }^{22}$, Kristen A. Panfilio ${ }^{8,23}$, Yoonseong Park ${ }^{24}$, Hugh M. Robertson ${ }^{25}$, Guy Smagghe ${ }^{4,13,14}$, Diane E. Ullman ${ }^{3}$, Maurijn van der Zee ${ }^{7}$, Thomas Van Leeuwen ${ }^{4}$, Jan A. Veenstra ${ }^{26}$, Robert M. Waterhouse ${ }^{27}$, Matthew T. Weirauch ${ }^{28,29}$, John H. Werren ${ }^{20}$, Anna E. Whitfield ${ }^{1}$, Evgeny M. Zdobnov ${ }^{6}$, Richard A. Gibbs ${ }^{16}$ and Stephen Richards ${ }^{16}$

\section{Correction to: BMC Biology 18, 142 (2020) https://doi.org/10.1186/s12915-020-00862-9}

Following publication of the original article [1], the authors would like to remove the phrase 'vertically transmitted' from the last sentence in the fourth paragraph under the heading Background.

The sentence originally read:

In addition to serving as crop disease vectors, thrips support vertically transmitted, facultative bacterial symbionts that reside in the hindgut $[27,28]$.

The sentence should read:

In addition to serving as crop disease vectors, thrips support facultative bacterial symbionts that reside in the hindgut $[27,28]$.

The original article [1] has been corrected.

The original article can be found online at https://doi.org/10.1186/s12915020-00862-9.

* Correspondence: drotenb@ncsu.edu

${ }^{1}$ Department of Entomology and Plant Pathology, North Carolina State University, Raleigh, NC 27695, USA

Full list of author information is available at the end of the article

\author{
Author details \\ 'Department of Entomology and Plant Pathology, North Carolina State \\ University, Raleigh, NC 27695, USA. ${ }^{2}$ Virology Section, College of Veterinary \\ Medicine, University of Tennessee, A239 VTH, 2407 River Drive, Knoxville, TN \\ 37996, USA. ${ }^{3}$ Department of Entomology and Nematology, University of \\ California Davis, Davis, CA 95616, USA. ${ }^{4}$ Laboratory of Agrozoology, \\ Department of Plants and Crops, Ghent University, Coupure Links 653, 9000 \\ Ghent, Belgium. ${ }^{5}$ Institute of Molecular Biology and Biotechnology, \\ Foundation for Research and Technology-Hellas, Vassilika Vouton, 70013 \\ Heraklion, Greece. ${ }^{6}$ Department of Genetic Medicine and Development, \\ University of Geneva Medical School, and Swiss Institute of Bioinformatics, \\ Geneva, Switzerland. ${ }^{7}$ Institute of Biology, Leiden University, 2333 BE Leiden, \\ The Netherlands. ${ }^{8}$ Institute for Zoology: Developmental Biology, University of \\ Cologne, 50674 Cologne, Germany. ${ }^{9}$ Department of Plant Pathology, \\ University of Georgia - Tifton Campus, Tifton, GA 31793-5737, USA. ${ }^{10}$ National \\ Agricultural Library, USDA-ARS, Beltsville, MD 20705, USA. ${ }^{11}$ Department of \\ Plant Pathology, Kansas State University, Manhattan, KS 66506, USA. \\ ${ }^{12}$ Department of Biology, University of Washington, Seattle, WA 98105, USA. \\ ${ }^{13}$ Chongqing Key Laboratory of Entomology and Pest Control Engineering, \\ College of Plant Protection, Southwest University, Chongqing, China. \\ ${ }^{14}$ International Joint Laboratory of China-Belgium on Sustainable Crop Pest \\ Control, Academy of Agricultural Sciences, Southwest University, Chongqing, \\ China and Ghent University, Ghent, Belgium. ${ }^{15}$ Department of Botany, \\ University of Ruhuna, Matara, Sri Lanka. ${ }^{16}$ Human Genome Sequencing \\ Center, Department of Human and Molecular Genetics, Baylor College of \\ Medicine, One Baylor Plaza, Houston, TX 77030, USA. ${ }^{17}$ Department of \\ Biological Sciences, University of Cincinnati, Cincinnati, OH 45221, USA.
}


${ }^{18}$ IPAVE-CIAP-INTA, 5020 Cordoba, Argentina. ${ }^{19}$ Department of Biology, Mount St. Joseph University, Cincinnati, OH 45233, USA. ${ }^{20}$ Department of Biology, University of Rochester, Rochester, NY 14627, USA. ${ }^{21}$ Queensland Alliance for Agriculture and Food Innovation, The University of Queensland, QLD, St. Lucia 4072, Australia. ${ }^{22}$ Department of Biological Sciences, Wayne State University, Detroit, MI 48202, USA. ${ }^{23}$ School of Life Sciences, University of Warwick, Gibbet Hill Campus, Coventry CV4 7AL, UK. ${ }^{24}$ Department of Entomology, Kansas State University, Manhattan, KS 66506, USA.

${ }^{25}$ Department of Entomology, University of Illinois at Urbana-Champaign, Urbana, IL 61801, USA. ${ }^{26}$ INCIA UMR 5287 CNRS, University of Bordeaux, Pessac, France. ${ }^{27}$ Department of Ecology and Evolution, Swiss Institute of Bioinformatics, University of Lausanne, 1015 Lausanne, Switzerland. ${ }^{28}$ Center for Autoimmune Genomics and Etiology, Divisions of Biomedical Informatics and Developmental Biology, Cincinnati Children's Hospital Medical Center, Cincinnati, OH 45229, USA. ${ }^{29}$ Department of Pediatrics, University of

Cincinnati, College of Medicine, Cincinnati, OH 45229, USA.

Published online: 16 November 2020

\section{Reference}

1. Rotenberg, et al. Genome-enabled insights into the biology of thrips as crop pests. BMC Biology. 2020;18:142 https://doi.org/10.1186/s12915-02000862-9.

Ready to submit your research? Choose BMC and benefit from:

- fast, convenient online submission

- thorough peer review by experienced researchers in your field

- rapid publication on acceptance

- support for research data, including large and complex data types

- gold Open Access which fosters wider collaboration and increased citations

- maximum visibility for your research: over $100 \mathrm{M}$ website views per year

At BMC, research is always in progress.

Learn more biomedcentral.com/submissions 